\title{
Design and Implementation of Conducted Emission Reference Source
}

\author{
Dongsheng Zhao, George Teunisse \\ VSL, Dutch Metrology Institute \\ Delft, The Netherlands \\ email: dzhao@vsl.nl
}

\begin{abstract}
To meet the requirement of doing periodic interlaboratory verification and self assessment, a device so called reference source is crucial. In this paper, an existing conducted emission reference source is reviewed and a new design is proposed. The considerations are explained, preliminary results are presented thereupon, and the benefits of the design are emphasized in the conclusion.
\end{abstract}

Keywords—design, conducted emission, reference source

\section{INTRODUCTION}

According to the ISO 17025 accreditation system periodic inter-laboratory verification and self assessment is required. In laboratories an emission reference source is used in order to provide a means for a quick and easy regular checking of the EMC emission test system, Such sources are commercial ly available now on the market applying various names . Names like: EMC test verification equipment [1], comb generator (CG) [2], comparison noise emitter (CNE) $[1,3]$, comb emitter [1], comb generator emitter [1], York reference source (YRS) [1], conducted reference source (CRS) $[4,5]$ etc. Even in some literature, when designed for research purposes these kinds of devices are referred to as generic test object (GTO) [6] and site source [7]. Often two types of measuring devices are distinguished between. The one used for testing the conducted emission test system: the so called "conducted emission reference source", the other being used to calibrate radiated emission test sites, referred to as "radiated emission reference source".

The radiated emission reference source is not involved in this investigation. The focus is on the conducted emission reference source which is referred to as "reference source" in the rest of this paper.

In test laboratories, all the test equipment requires regular calibration, but the calibration is generally performed on a remote location. When test equipment is integrated in a measurement system, verification is necessary between calibrations while there will be no guarantee that all connectors will stay correctly and firmly connected in the mean time, or that test equipment will not show any malfunctioning. This concerns the aspect of a hardware fault. The other aspect concerned is software faults, for instance, incorrect settings of equipment or wrong operation procedures.

This work is funded by the European Union on the basis of Decision No 912/2009/EC, and identified in the European Metrology Research Program (EMRP) as Joint Research Project (JRP) IND60 EMC, Improved EMC test methods in industrial environments. Additional funding was received from the EMRP participating countries.

\author{
Frank Leferink \\ University of Twente, \\ Enschede, The Netherlands \\ email: leferink@ieee.org
}

The reference source plays the role of verification standard. It may also be used for inter-laboratory round-robin test. Although this way of verification is part of their accreditation procedures, at present, for the EMC test laboratories this is often very difficult to fulfill.

In this paper, the existing conducted emissions reference source is reviewed and a new design is proposed. The considerations are explained, preliminary results are presented thereupon, and the benefits of the design are emphasized in the conclusions.

\section{DESIGN}

In order to fulfill its purpose, a reference source should be sufficiently stable. As a rule of thumb its fluctuation should be 2-5 times smaller than the uncertainty of the measurement system. A typical measurement uncertainty for a full compliance conducted emissions test to EN 55022 or EN 55011 would be $\pm 2.5 \mathrm{~dB}$. That means that the reference source should provide $\pm 0.5 \mathrm{~dB}$ stability in all circumstances. This requirement is not yet satisfied at present.

The reference source should be applicable for an as wide as possible range of test standards. Most standards concern EMI levels in the frequency range $150 \mathrm{kHz}-30 \mathrm{MHz}$. For electronic lighting devices, the limits on conducted emissions start at $9 \mathrm{kHz}$. Since recently the EMI issue between $2 \mathrm{kHz}$ and $150 \mathrm{kHz}$ is being widely concerned [9], this frequency range $2 \mathrm{kHz}-150 \mathrm{kHz}$ should also be covered. Another enhancement is to extend the upper frequency range to 300 MHz. For instance, EN55015 for luminaries was changed such that radiated emission testing between $30 \mathrm{MHz}-$ $300 \mathrm{MHz}$ now is required to be performed by applying a conducted emission test method. Therefore, the reference source should cover the following three CISPR ranges.

TABLE I. REFERENCE SOURCE FREQUENCY RANGES

\begin{tabular}{|c|c|c|}
\hline \multicolumn{3}{|c|}{ Frequency ranges } \\
\hline Band A & Band B & Band C \\
$2-150 \mathrm{kHz}$ & $0.15-30 \mathrm{MHz}$ & $30-300 \mathrm{MHz}$ \\
\hline
\end{tabular}

In addition, the output levels of the reference source should not be fixed values as in the previous designs. The output levels should be adjustable and be as close as possible 
to the defined limit lines. This is based on the following considerations:

- At present, reference sources cannot be used without having a data table that contains its output signal level at each frequency point. Such calibration data normally is provided by the reference source manufacturer. It is tedious and is very likely to make a mistake when comparing the measurement results with the data in the data table. In the new design, the output signal of the reference source is set to the limit lines values and the verification is done by comparing the measured value with the limit lines, just like performing normal measurements.

- Another argument why a reference source should provide an emission level close to limit line can be explained by the following example. An inexperienced operator uses an under-sized amplifier or limiter in a measurement setup. In the beginning, he has checked the setup using a reference source which emits only low level signal. The verification is done successfully. But when the real measurement is performed, due to the high emission level, the result gets a large deviation. In such a situation, this formerly design reference source cannot fulfill the verification task correctly.

- Most of the EMC measurement results are judged close to the limit lines as shown in Fig. 1. It makes sense to avoid the influence of non-linearity of equipment by performing verification as close as possible to the levels defined by the limit lines.

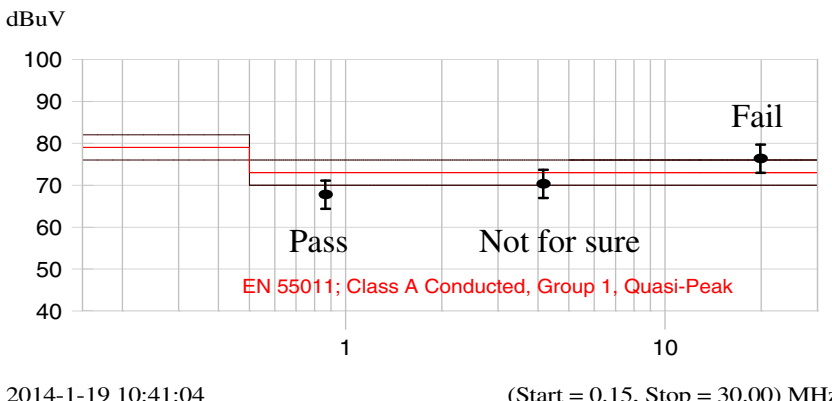

Fig. 1. EMC are judged close to limit lines

Most of the existing reference sources are using a crystal oscillator module as the digital pulse train source. The digital pulses are then divided and reshaped into fast changing pulses using Schmitt triggering. The pulse signal can be approximated by trapezoidal waveforms having $50 \%$ duty cycle while the waveforms have some non-zero rise time, and a non-zero fall time. The rise and fall times determine the amount of harmonic content in the frequency domain.

The harmonic having the lowest frequency is the fundamental frequency. Its level is $2 \mathrm{~A} / \pi$, where $\mathrm{A}$ is the peak-peak amplitude of the trapezoidal pulse. Assume A is $5 \mathrm{~V}$, for $50 \%$ duty cycle trapezoidal signal, the level of the fundamental frequency then is $10 / \pi \mathrm{V}$, which is about 130
$\mathrm{dB} \mu \mathrm{V}$. For the frequency beyond the fundamental frequency, the spectrum's envelope will have a slope of $-20 \mathrm{~dB}$ per decade from the fundamental to that frequency.

The existing reference sources have the following limitations:

The shape and amplitude of the harmonics are difficult to be manipulated. The limit lines in different standards have different levels and different slopes as shown in Fig. 2. Their levels can vary in a range of $50 \mathrm{~dB}$. Some of them have a slope of $20 \mathrm{~dB} /$ decade, some have flat limit lines or a slope of $25.7 \mathrm{~dB} /$ decade (EN55011 in frequency range $5 \mathrm{MHz}-30$ $\mathrm{MHz}$ ). By changing the rise and fall times, the spectrum above $1 / \pi \tau_{r}$ is influenced, also the corner frequency $1 / \pi \tau_{r}$ changes, but the spectrum below the corner frequency is not changed.

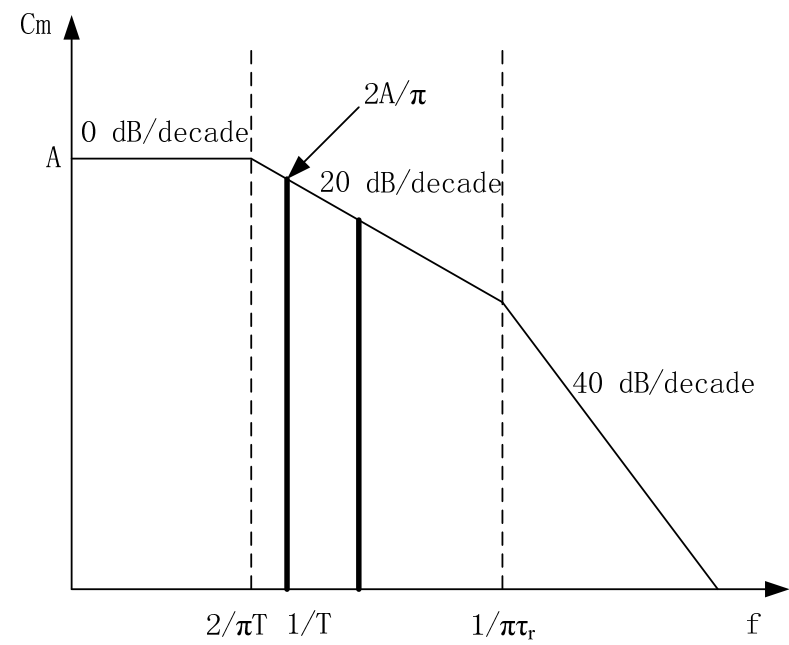

Fig. 2. The envelop of the spectrum of trapezoidal waveforms with $50 \%$ duty cycle

Some existing reference sources pass the square wave through a filter to reshape the amplitude level of subsequent harmonics, having a slope of $20 \mathrm{~dB} / \mathrm{dec}$ ade, to an almost equal amplitude level. Changing the amplitude of a pulse could also be performed by adjusting amplitudes of all harmonics together, but it would be very difficult to have a reference source generating the signal spectrum following the shape and level of limit lines in this manner.

It is difficult to find a suitable pulse repetition frequency (PRF). If a high PRF is chosen, for instance $1 \mathrm{MHz}$, nice harmonics up to $300 \mathrm{MHz}$ or higher can be produced, but there will be too sparse reference points provided in the lower frequencies.

Using a low PRF for the whole frequency range is also not ideal. For instance, if the PRF is set to $1 \mathrm{kHz}$, the level of the fundamental harmonics at $1 \mathrm{kHz}$ must be at least 160 $\mathrm{dB} \mu \mathrm{V}$ to obtain $70 \mathrm{~dB} \mu \mathrm{V}$ in $30 \mathrm{MHz}$. This is not realistic. 


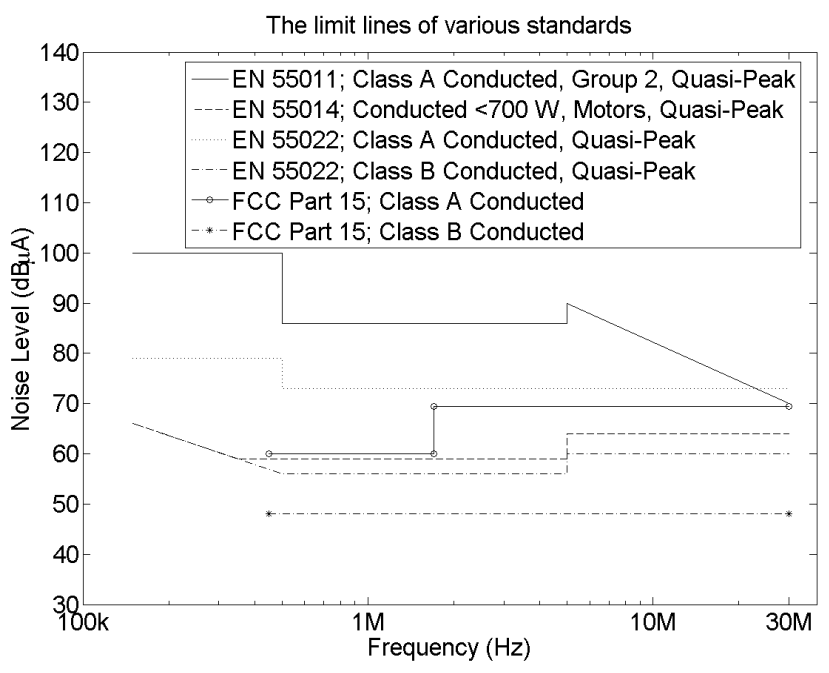

Fig. 3. The limit lines in various standards

Based on the above considerations, the design of the reference source is split up in three segments. The PRF is chosen to be 5-20 times higher than the receiver bandwidth. This is with the purpose to avoid the detector response correction due to the low PRF.

TABLE II. THE RECEIVER BANDWIDTH AND THE FUNDAMENTAL FREQUENCY OF THE REFERENCE SOURCE

\begin{tabular}{|c|c|c|c|}
\hline & \multicolumn{3}{|c|}{ Frequency band } \\
\hline & $\begin{array}{c}\text { Band A } \\
9-150 \mathrm{kHz}\end{array}$ & $\begin{array}{c}\text { Band B } \\
0.15-30 \\
\mathrm{MHz}\end{array}$ & $\begin{array}{c}\text { Band C } \\
30-300 \mathrm{MHz}\end{array}$ \\
\hline $\begin{array}{c}6 \mathrm{~dB} \\
\text { bandwidth }\end{array}$ & $200 \mathrm{~Hz}$ & $9 \mathrm{kHz}$ & $120 \mathrm{kHz}$ \\
\hline $\begin{array}{c}\text { Fundamental } \\
\text { frequency }\end{array}$ & $1 \mathrm{kHz}$ & $150 \mathrm{kHz}$ & $10 \mathrm{MHz}$ \\
\hline $\begin{array}{c}\text { Waveform } \\
\text { composition }\end{array}$ & $\begin{array}{c}1-150 \\
\text { harmonics }\end{array}$ & $\begin{array}{c}1-200 \\
\text { harmonics }\end{array}$ & $\begin{array}{c}1-31 \text { odd } \\
\text { harmonics }\end{array}$ \\
\hline
\end{tabular}

The generator is divided in three segments based on the following considerations:

1. In the band A and B, because this frequency band is relatively low, it is possible to generate the desired waveform using a D/A converter. The signal is generated using the direct digital synthesizer (DDS) method. This provides flexibility to generate the signal having the desired spectrum, because the level of each harmonic is independently adjustable.

2. Different fundamental frequencies are used to achieve a sufficient number of frequency points in the band $\mathrm{A}$ and sufficient emission level in the band B.

3. For the band $\mathrm{C}$, it is not easy to find any arbitrary waveform generator which can cover the whole frequency range. Therefore the oscillator method needs to be applied. When using the low pulse repetition frequency, the signal level will decrease such that it will level below the noise floor. Therefore, a higher pulse repetition frequency (10 $\mathrm{MHz}$ ) is used. Here a capacitor is used to isolate the dc component of reference source. The capacitor further also reshapes the output harmonics level to $0 \mathrm{~dB} /$ decade.

In the realization of DDS method, already the amplitude requirements of each harmonic is known, but the phase of each frequency components is still not defined. Using the trapezoidal signal as the noise source is actually aligning all frequency components in the rise and fall edge. It is however not yet the optimized waveform. Since by using the DDS method an arbitrary waveform can be composited, it offers the generation of a signal having the lowest crest factor, so that herewith the dynamic range of the D/A converter can be extended. This way of approach is applying the method developed by Schroeder [10].

Consider the periodic signal $s(t)$ with period $T$ is composited of the $N$ harmonics.

$$
s(t)=\sum_{n=1}^{N} h_{n} \cos \left(\frac{2 \pi n t}{T}+\theta_{n}\right)
$$

First, the desired amplitudes of each harmonic $h_{n}$ are calculated considering the limit lines and compensation of the non-flatness of the waveform generator.

Then the relative power $p_{n}$ of the $n$-th harmonic is calculated:

$$
p_{n}=\frac{h_{n}^{2}}{\sum_{k=1}^{N} h_{k}^{2}}
$$

The $\theta_{k}$ in (1) is calculated using (3)

$$
\theta_{n}=\theta_{1}-2 \pi \sum_{k=1}^{n-1}(n-k) p_{k} \quad \text { for } n \geq 2
$$

$\theta_{1}$ is set to a fix value, for instance, $-\pi / 2$.

Herewith the desired phase angles of each of the harmonics are obtained. These are used to generate the waveform having the smallest crest factor.

Fig. 4 below shows the deviations with and without phase optimization. In this example, the peak to peak amplitude is reduced 2.61 times, by adjusting the phase setting. For signal composited of hundreds of harmonics, the improvement may be as large as 5 times. 


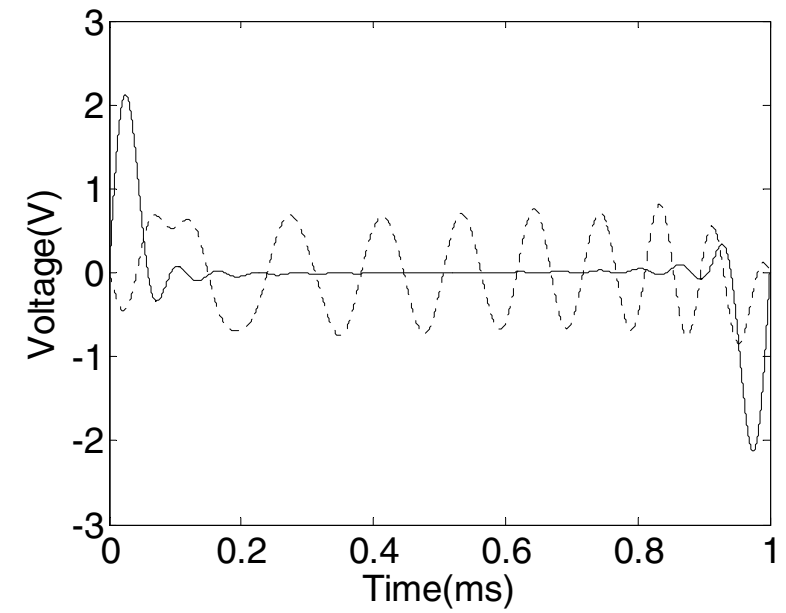

Fig. 4. One period of two signals composited of 16 harmonics with the same power spectrum. For the solid line curve, all phase angles equal to $-\pi / 2$. For dotted curve, the phase angles have been optimized according to (3).

\section{IMPLEMENTATION}

A commercial off-the-shelf product has been used to show the feasibility of the design. The signal is generated using an arbitrary waveform generator (AWG), model Tektronix AFG3102 ${ }^{1}$. Its specifications are:

-14-bit vertical resolution

- $1 \mathrm{GSa} / \mathrm{s}, 16 \mathrm{k}$-point resolution arbitrary waveforms

- Typical rise/fall time $8 \mathrm{~ns}$

- $50 \mathrm{MHz}$ synthesized function generator

The data is prepared in Matlab and sent to the AWG using ArbExpress software. The waveform parameters are set to designed values.

In CISPR band $\mathrm{C}$, the AWG will drive a diode, being a Schottky or step recovery diode (SRD) when the rise and fall time of the AWG is not satisfying. It generates discrete frequencies spaced $10 \mathrm{MHz}$ apart in the frequency range 30 $\mathrm{MHz}-300 \mathrm{MHz}$.

\section{PRELIMINARY RESULT}

In the following, the measurement results of the spectrum of the reference source are shown.

The first measured waveform contains the fundamental frequency and harmonics up to $150^{\text {th }}$ order. In this measurement, all harmonics are set to the same level. The fundamental frequency is set to $1 \mathrm{kHz}$. In Fig. 5 the spectrum of the reference source in band $\mathrm{A}$ is shown. The amplitude flatness in the lower frequency range is quite satisfactory.

\footnotetext{
${ }^{1}$ The manufacturers and types of instrumentation mentioned in this paper do not indicate any preference by the authors, nor does it indicate that these are the best available for the application discussed.
}

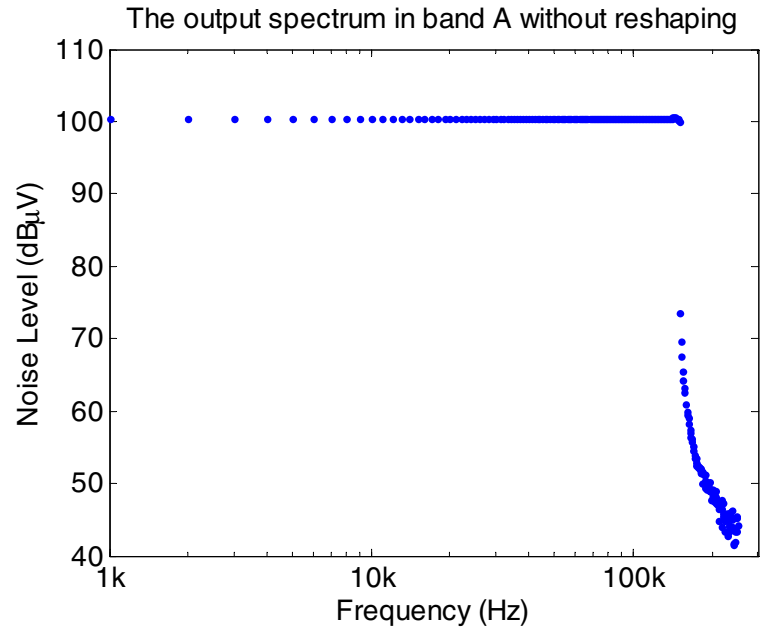

Fig. 5. The spectrum of the reference source in band A

The second measured waveform contains harmonics from $150 \mathrm{kHz}$ to $30 \mathrm{MHz}\left(1^{\text {st }}-200^{\text {th }}\right.$ harmonics $)$. In this measurement, all harmonics are set to the same level, but the fluctuation of all harmonics beyond $12 \mathrm{MHz}$ is observed. Therefore it is necessary to compensate for this non flatness using the pre-distortion method.

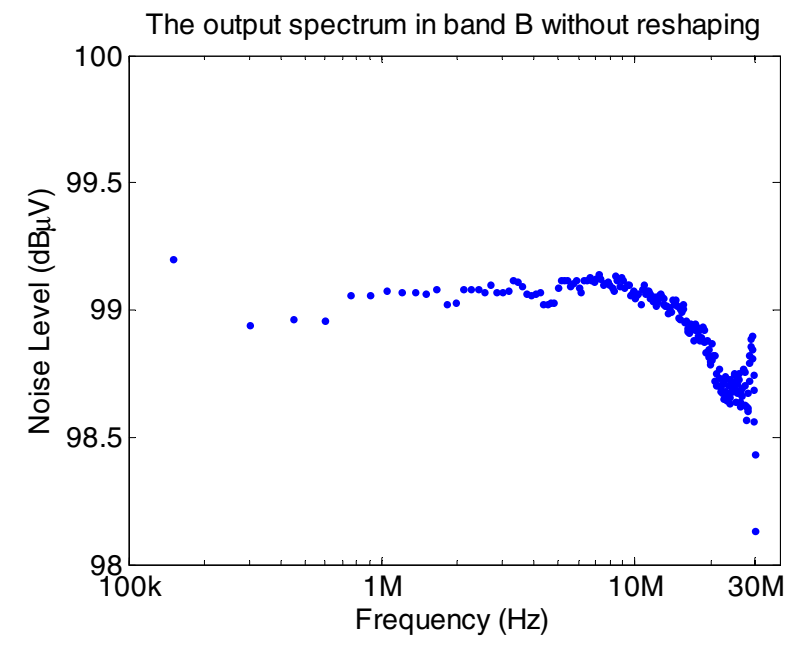

Fig. 6. The output spectrum of the reference source in band B without reshaping

The non flatness in the higher frequency range is compensated and the level of each harmonic is now set close to the limit lines required by EN 55011 Class A: Conducted emission using $Q P$ detector. The measurement result is shown in Fig. 7 and the limit line from the standard is shown in Fig. 8. 


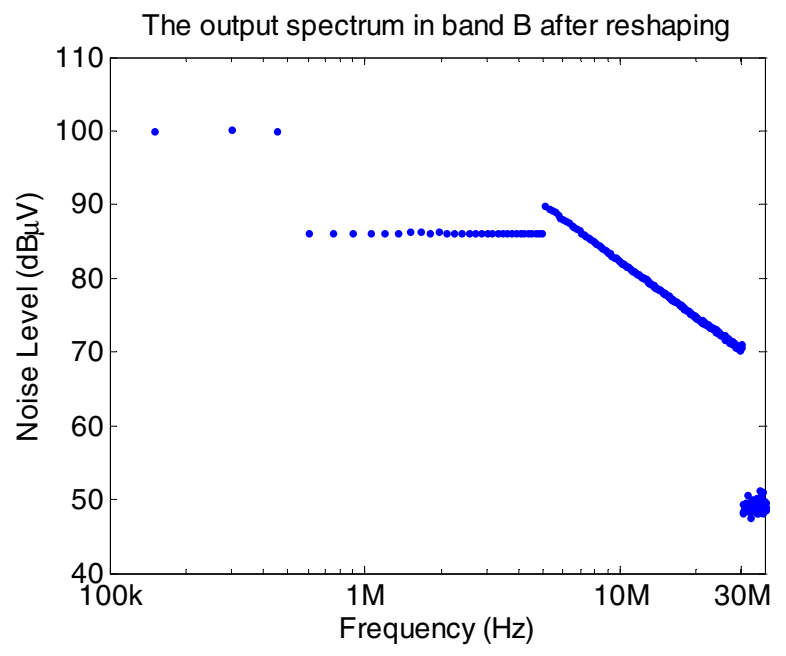

Fig. 7. The output spectrum of the reference source in band B after reshaping

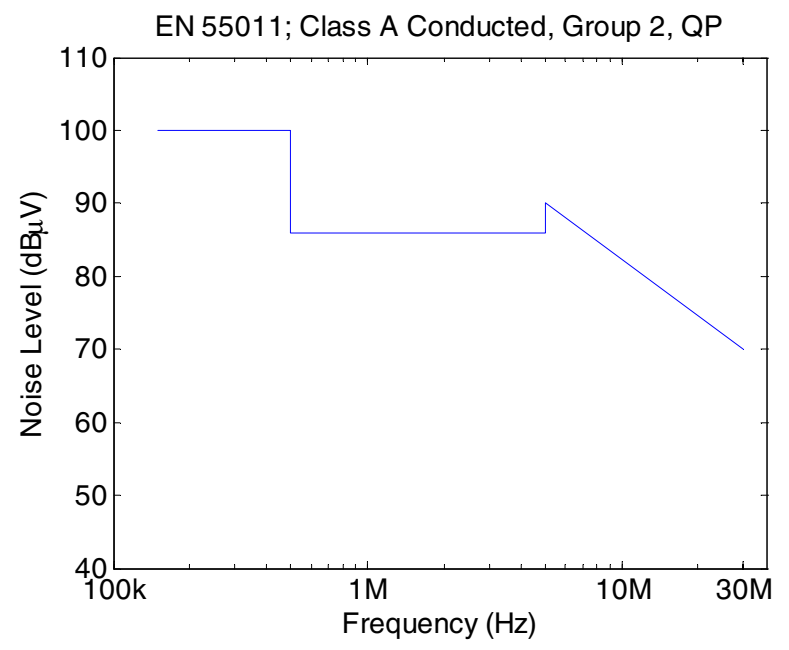

Fig. 8. The limit line of EN 55011; Class A Conducted emission, QP detector in band B

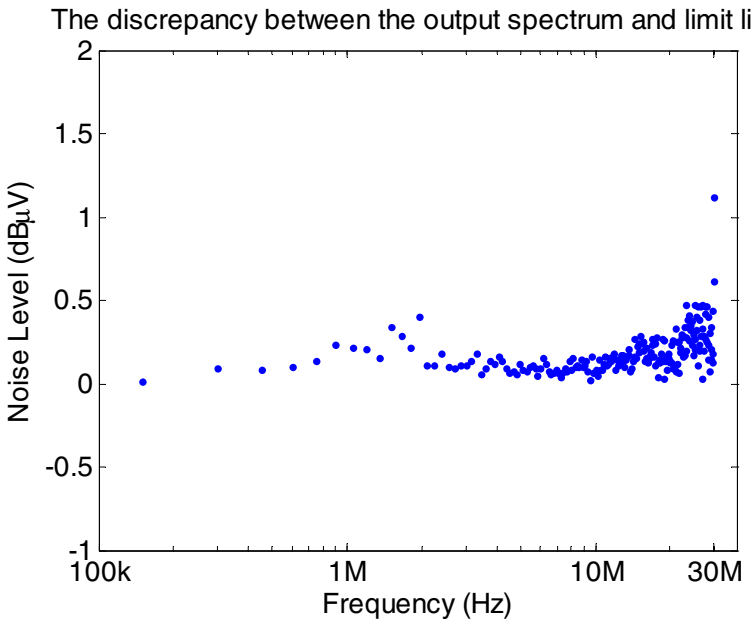

Fig. 9. The discrepancy between the output spectrum and the limit line
By comparing Fig. 7 and Fig. 8, the discrepancy between the output spectrum and limit line is shown in Fig 9. The preset requirement is satisfied except for a few points around $30 \mathrm{MHz}$. The emission levels of each of the harmonics of the reference source are controllable and close to the limit line.

The stability of the reference source was measured using the zero-frequency span method of the spectrum analyzer. The result is shown in Fig. 10. The fluctuation stays within $0.02 \mathrm{~dB}$ in $1600 \mathrm{~s}$.

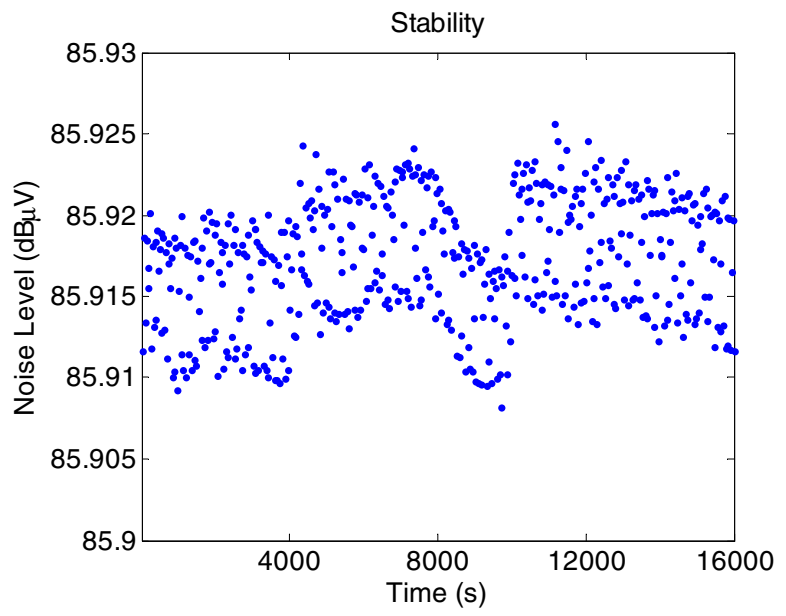

Fig. 10. The emission level in a timespan of 16000 seconds of the reference source in band B, measured using a spectrum analyzer in zero frequency span mode and $3.9 \mathrm{MHz}$ central frequency.

The third measured waveform contains the fundamental frequency $10 \mathrm{MHz}$ and harmonics up to $300 \mathrm{MHz}$. Its spectrum is shown in Fig. 11.

In this frequency range, the spectrum is not controllable. The emission level of harmonics is determined by the characteristics of components, mainly the edges of reverse recovery current of the diode. The harmonic levels cannot be adjusted to a predefined value.

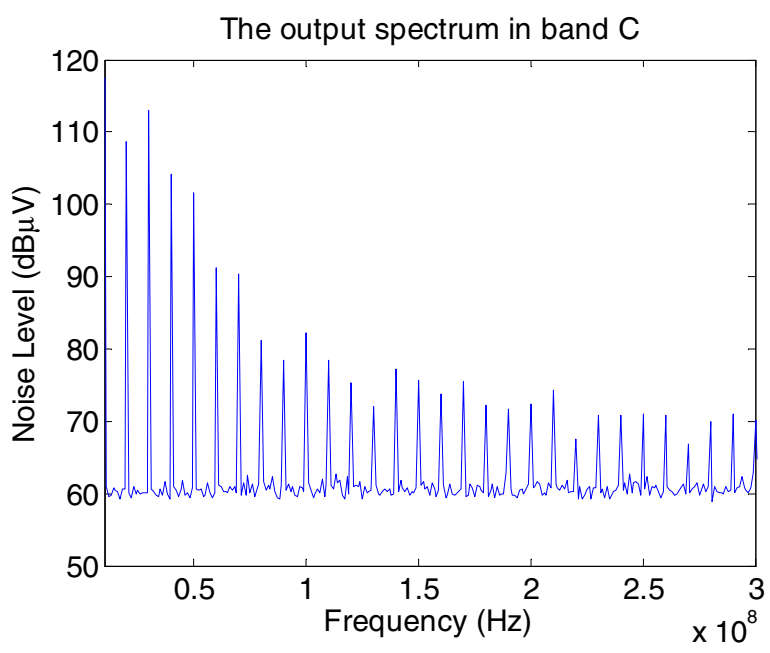

Fig. 11. The output spectrum of the reference source in band $\mathrm{C}$ 


\section{CONCLUSIONS}

Using a simple crystal oscillator module and a coupling capacitor and connecting the output to a coax connector is the principle of most existing commercial reference sources. This kind of comb generator is cheap, simple but has some limitations.

In this presented new design, an AWG is used to be the reference source in CISPR band A and B. The level of each harmonic is adjustable. Special attention is paid to the phase setting of harmonics so that the crest factor of the waveform is minimized. In the CISPR band C, the oscillator method is applied. The AWG generates a rectangular waveform or drives a step recovery diode (SRD) as reference source if the rise and fall time of the AWG is not satisfying. By combining advantages of these two methods, this reference source becomes a useful verification kit and transfer standard for EMC test laboratories.

\section{REFERENCES}

[1] York EMC Services Ltd, Available:

http://www.yorkemc.co.uk/instrumentation/.
[2] COM-POWER, Available:

http://www.com-power.com/comb_generators.html

[3] AR, Available:

http://www.arww-rfmicro.com/html/18200.asp?id=229

[4] Comtest, Available:

http://www.emc-partner.fr/index_fichiers/Datasheets/Cages-DiversCOMTEST/1410\%20FRS.pdf

[5] Laplace, Available:

http://www.laplace.co.uk/media/download/CRSmanual.pdf

[6] TEMCA2, Available: http://www2.fhi.nl/praktijkdag/archief/2010/images/khbo1.pdf

[7] D.-C. Chang, C.-H. Lee and T. Y. Yang, "Novel Design of Site Source for Radiation and Conduction Emission Test", Progress In Electromagnetics Research Symposium Proceedings, pp. 1033-1035, 2013

[8] J. Bernal and V. Ungvichian, "A Study of Conducted-Emission Stable Source Applied to the EMC US and EU Standards", in proc. International Latin American and Caribbean Conference for Engineering and Technology Conference (LACCEI), pp. IT042, 2006.

[9] E. O. A Larsson and M. H. J. Bollen, "Emission and Immunity of Equipment in the Frequency Range 2 to $150 \mathrm{kHz}$ ", in proc. IEEE Bucharest Power Tech Conference, 2009.

[10] M.R. Schroeder, "Synthesis of Low-Peak-Factor Signals and Binary Sequences with low Autocorrelation," IEEE Trans. Information Theory, vol. 16, pp.85-89, January 1970 